\title{
A Note on the Triple Laplace Transform and Its Applications to Some Kind of Third-Order Differential Equation
}

\begin{abstract}
Abdon Atangana
Institute for Groundwater Studies, Faculty of Natural and Agricultural Sciences, University of the Free State, Bloemfontein 9300, South Africa

Correspondence should be addressed to Abdon Atangana; abdonatangana@yahoo.fr

Received 25 March 2013; Accepted 20 May 2013

Academic Editor: R. K. Bera

Copyright (C) 2013 Abdon Atangana. This is an open access article distributed under the Creative Commons Attribution License, which permits unrestricted use, distribution, and reproduction in any medium, provided the original work is properly cited.

We introduced a relatively new operator called the triple Laplace transform. We presented some properties and theorems about the relatively new operator. We examine the triple Laplace transform of some function of three variables. We make use of the operator to solve some kind of third-order differential equation called "Mboctara equations."
\end{abstract}

\section{Introduction}

The topic of partial differential equations is one of the most important subjects in mathematics and other sciences. The behaviour of the solution very much depends essentially on the classification of PDEs therefore the problem of classification for partial differential equations is very natural and well known since the classification governs the sufficient number and the type of the conditions in order to determine whether the problem is well posed and has a unique solution. The Laplace transform has been intensively used to solve nonlinear and linear equations [1-7]. The Laplace transform is used frequently in engineering and physics; the output of a linear time invariant system can be calculated by convolving its unit impulse response with the input signal. Performing this calculation in Laplace space turns the convolution into a multiplication; the latter is easier to solve because of its algebraic form. The Laplace transform can also be used to solve differential equations and is used extensively in electrical engineering [1-7]. The Laplace transform reduces a linear differential equation to an algebraic equation, which can then be solved by the formal rules of algebra. The original differential equation can then be solved by applying the inverse Laplace transform. The English electrical engineer Oliver Heaviside first proposed a similar scheme, although without using the Laplace transform, and the resulting operational calculus is credited as the Heaviside calculus. Recently Kılıçman et al. [8-11] extended the Laplace transform to the concept of double Laplace transform. This new operator has been intensively used to solve some kind of differential equation [11] and fractional differential equations. The aim of this work is to extend the Laplace transform to the triple Laplace transform. We will start with the definition of the triple Laplace transform.

\section{Definitions and Theorems}

Definition 1. Let $f$ be a continuous function of three variables; then, the triple Laplace transform of $f(x, y, t)$ is defined by

$$
\begin{aligned}
& L_{x, y, t}[f(x, y, t)] \\
&=F(p, s, k) \iiint_{0}^{\infty} \exp [-p x] \exp [-s y] \\
& \quad \times \exp [-k t] f(x, y, t) d x d y d t,
\end{aligned}
$$

where, $x, y, t>0$ and $p, s, k$ are Laplace variables, and

$$
\begin{aligned}
& f(x, y, t) \\
& =\frac{1}{2 \pi i} \int_{\alpha-i \infty}^{\alpha+i \infty} e^{p x} \\
& \times\left[\frac{1}{2 \pi i} \int_{\beta-i \infty}^{\beta+i \infty} e^{s y}\right.
\end{aligned}
$$




$$
\begin{aligned}
& \times\left[\frac{1}{2 \pi i} \int_{\mu-i \infty}^{\mu+i \infty} e^{k t}\right. \\
& \quad \times F(p, s, k) d k] d s] d p
\end{aligned}
$$

is the inverse triple Laplace transform.

Property 2. Assuming that the continuous function $f(x, y, t)$ is triple Laplace transformable, then,

$$
\begin{aligned}
& L_{t, y, x}[\left.\frac{\partial^{3} f(x, y, t)}{\partial x \partial y \partial t}\right] \\
&= p s k F(p, s, k)-p s F(p, s, 0)-p s F(p, 0, k) \\
&+p F(p, 0,0)-s k F(0, s, k)+s F(0, s, 0) \\
&+k F(0,0, k)-F(0,0,0), \\
& L_{x, x, t}[\left.\frac{\partial^{3} f(x, y, t)}{\partial t \partial x^{2}}\right] \\
&= k p^{2} F(p, y, k)-p k F(0, y, k)-\frac{\partial F(0, y, k)}{\partial x} \\
&-p^{2} F(p, y, 0)+p F(0, y, 0)+\frac{\partial F(0, y, 0)}{\partial x}, \\
& L_{x x x}\left[\begin{array}{ll}
\partial^{3} f(x, y, t) \\
\partial x^{3}
\end{array}\right] \\
&= p^{3} F(p, y, t)-p^{2} F(0, y, t) \\
&-p \frac{\partial F(0, y, t)}{\partial x}-\frac{\partial^{2} F(0, y, t)}{\partial x^{2}} .
\end{aligned}
$$

\section{Uniqueness and Existence of the Triple Laplace Transform}

In this section, we will study the uniqueness and existence of triple Laplace transform. First of all, let $f(x, y, t)$ be a continuous function on the interval $[0, \infty)$ which is of exponential order, that is, for some $a, b, c \in R$. Consider

$$
\sup _{x, y, t>0}\left|\frac{f(x, y, t)}{\exp [a x+b y+c t]}\right|<0 .
$$

Under the previous condition, the triple Laplace transform,

$$
\begin{aligned}
F(p, s, k)=\iiint_{0}^{\infty} \exp [-p x] \exp [-s y] \\
\quad \times \exp [-k t] f(x, y, t) d x d y d t,
\end{aligned}
$$

exists for all $p>a, s>b$, and $k>c$ and is in actuality infinitely differentiable with respect to $p>a, s>b$ and $k>c$. All functions in this study are assumed to be of exponential order. The following theorem shows that $f(x, y, t)$ can be uniquely obtained from $F(p, s, t)$.
Theorem 3. Let $f(x, y, t)$ and $g(x, y, t)$ be continuous functions defined for $x, y, t \geq 0$ and having Laplace transforms, $F(p, s, k)$ and $G(p, s, k)$, respectively. If $F(p, s, k)=G(p, s, k)$, then $f(x, y, t)=g(x, y, t)$.

Proof. From the definition of the inverse Laplace transform, if $\alpha, \beta$, and $\mu$ are sufficiently large, then the integral expression, by

$$
\begin{aligned}
& f(x, y, t) \\
& =\frac{1}{2 \pi i} \int_{\alpha-i \infty}^{\alpha+i \infty} e^{p x} \\
& \times\left[\frac{1}{2 \pi i} \int_{\beta-i \infty}^{\beta+i \infty} e^{s y}\right. \\
& \times\left[\frac{1}{2 \pi i} \int_{\mu-i \infty}^{\mu+i \infty} e^{k t}\right. \\
& \quad \times F(p, s, k) d k] d s] d p,
\end{aligned}
$$

for the triple inverse Laplace transform, can be used to obtain

$$
\begin{aligned}
& f(x, y, t) \\
& =\frac{1}{2 \pi i} \int_{\alpha-i \infty}^{\alpha+i \infty} e^{p x} \\
& \times\left[\frac{1}{2 \pi i} \int_{\beta-i \infty}^{\beta+i \infty} e^{s y}\right. \\
& \times\left[\frac{1}{2 \pi i} \int_{\mu-i \infty}^{\mu+i \infty} e^{k t}\right. \\
& \times F(p, s, k) d k] d s] d p .
\end{aligned}
$$

By hypothesis, we have that $F(p, s, k)=G(p, s, k)$. then replacing this in the previous expression, we have the following:

$$
\begin{aligned}
& f(x, y, t) \\
& =\frac{1}{2 \pi i} \int_{\alpha-i \infty}^{\alpha+i \infty} e^{p x} \\
& \times\left[\frac{1}{2 \pi i} \int_{\beta-i \infty}^{\beta+i \infty} e^{s y}\right. \\
& \times\left[\frac{1}{2 \pi i} \int_{\mu-i \infty}^{\mu+i \infty} e^{k t}\right. \\
& \times G(p, s, k) d k] d s] d p,
\end{aligned}
$$


which boil down to

$$
\begin{aligned}
& f(x, y, t) \\
& =\frac{1}{2 \pi i} \int_{\alpha-i \infty}^{\alpha+i \infty} e^{p x} \\
& \times\left[\frac{1}{2 \pi i} \int_{\beta-i \infty}^{\beta+i \infty} e^{s y}\right. \\
& \times\left[\frac{1}{2 \pi i} \int_{\mu-i \infty}^{\mu+i \infty} e^{k t}\right. \\
& \times G(p, s, k) d k] d s] d p, \\
& =g(x, y, t) \text {, }
\end{aligned}
$$

and this proves the uniqueness of the triple Laplace transform.

Theorem 4. If, at the point $(p, s, k)$, the integrals

$$
\begin{aligned}
& F_{1}(p, s, k)=\iiint_{0}^{\infty} \exp [-p x] \exp [-s y] \\
& \times \exp [-k t] f_{1}(x, y, t) d x d y d t \\
& F_{2}(p, s, k)=\iiint_{0}^{\infty} \exp [-p x] \exp [-s y] \\
& \times \exp [-k t] f_{2}(x, y, t) d x d y d t
\end{aligned}
$$

are convergent and in addition if

$$
\begin{aligned}
F_{3}(p, s, k)=\iiint_{0}^{\infty} & \exp [-p x] \exp [-s y] \\
& \times \exp [-k t] f_{3}(x, y, t) d x d y d t
\end{aligned}
$$

is absolutely convergent, then, the following expression:

$$
F(p, s, k)=F_{1}(p, s, k) F_{2}(p, s, k) F_{3}(p, s, k)
$$

is the Laplace transform of the function

$$
\begin{gathered}
f(x, y, t) \\
=\int_{0}^{t} \int_{0}^{y} \int_{0}^{x} f_{3}\left(x-\left(x_{1}+\rho\right), y-\left(y_{1}+\sigma\right)\right. \\
\left.t-\left(t_{1}+\tau\right)\right) f_{2}\left(x_{1}-\rho, y_{1}-\sigma, t_{1}-\tau\right) \\
\quad \times f_{1}(\rho, \sigma, \tau) d \rho d \sigma d \tau
\end{gathered}
$$

and the integral

$$
\begin{aligned}
F(p, s, k)=\iiint_{0}^{\infty} \exp [-p x] \exp [-s y] \\
\quad \times \exp [-k t] f(x, y, t) d x d y d t
\end{aligned}
$$

is convergent at the point $(p, s, k)$; for the readers who are interested, they can see the proof in $[11,12]$.
Theorem 5. A function $f(x, y, t)$ which is continuous on $[0, \infty)$ and satisfies the growth condition (4) can be recovered from only $F(p, s, k)$ as

$$
\begin{aligned}
f(x, y, t)= & \lim _{\substack{n_{1} \rightarrow \infty \\
n_{2} \rightarrow \infty \\
n_{3} \rightarrow \infty}} \frac{(-1)^{n_{1}+n_{2}+n_{3}}}{n_{1} ! n_{2} ! n_{3} !}\left(\frac{n_{1}}{x}\right)^{n_{1}+1}\left(\frac{n_{2}}{y}\right)^{n_{2}+1} \\
& \times\left(\frac{n_{3}}{t}\right)^{n_{3}+1} \mathrm{X}^{n_{1}+n_{2}+n_{3}}\left[\frac{n_{1}}{x}, \frac{n_{2}}{y}, \frac{n_{3}}{t}\right] .
\end{aligned}
$$

Evidently, the main difficulty in using Theorem 5 for computing the inverse Laplace transform is the repeated symbolic differentiation of $F(p, s, k)$.

Let us see how Theorem 5 can be applicable. Let us consider the following functions:

$$
f(x, y, t)=\exp [-a x-b y-c t] .
$$

Naturally the triple Laplace transform of the previous function is given later as

$$
F(p, s, k)=\frac{1}{(p-a)(s-b)(k-c)} .
$$

Now applying the high-order mixed derivative to the previous expression, we obtain the following:

$$
\begin{aligned}
\frac{\partial^{n_{1}+n_{2}+n_{3}}[F(p, s, k)]}{\partial p^{n_{1}} \partial s^{n_{2}} \partial k^{n_{3}}}= & n_{1} ! n_{2} ! n_{3} !(-1)^{n_{1}+n_{2}+n_{3}} \\
& \times(a+P)^{-1-n_{1}}(s+b)^{-1-n_{2}}(c+k)^{-1-n_{3}} .
\end{aligned}
$$

Applying Theorem 5 in the previous expression, we obtain the following result:

$$
\begin{aligned}
f(x, y, t)= & \lim _{\substack{n_{1} \rightarrow \infty \\
n_{2} \rightarrow \infty \\
n_{3} \rightarrow \infty}} \frac{n_{1}{ }^{1+n_{1}} n_{2}{ }^{1+n_{2}} n_{3}{ }^{1+n_{3}}}{x^{n_{1}+1} y^{n_{2}+1} t^{n_{3}+1}}\left(a+\frac{n_{1}}{x}\right)^{-n_{1}-1} \\
& \times\left(b+\frac{n_{2}}{y}\right)^{-n_{2}-1}\left(c+\frac{n_{3}}{t}\right)^{-n_{3}-1} .
\end{aligned}
$$

Making a change of variable in the previous expression, we obtain the following simplified result:

$$
\begin{aligned}
f(x, y, t)= & \lim _{\substack{n_{1} \rightarrow \infty \\
n_{2} \rightarrow \infty \\
n_{3} \rightarrow \infty}}\left(1+\frac{a n_{1}}{x}\right)^{-n_{1}-1}\left(1+\frac{b n_{2}}{y}\right)^{-n_{2}-1} \\
& \times\left(1+\frac{c n_{3}}{t}\right)^{-n_{3}-1} .
\end{aligned}
$$

Using together, the application of logarithm and the L'Hôpital's rule on the previous expression, we arrive at the following result:

$$
\begin{aligned}
\ln (f(x, y, t)) & =-a x-b y-c t \Longrightarrow f(x, y, t) \\
& =\exp [-a x-b y-c t] .
\end{aligned}
$$




\section{Some Properties of Triple Laplace Transform}

In this section, we present some properties of the triple Laplace transform. Note that these properties follow from those of the double Laplace transform introduced by Kılıçman and Eltayeb [8]. The properties of the triple Laplace transform will enable us to find further transform pairs $\{f(x, y, t), F(p, s, k)\}$ :

$$
\text { (i) } \begin{aligned}
F(p+a, s+b, k+d) \\
=L_{x, y, t}\left[e^{-a x-y b-c t} f(x, y, t)\right](p, s, k) .
\end{aligned}
$$

We will present the proof

$$
\begin{aligned}
& L_{x, y, t}\left[e^{-a x-y b-c t} f(x, y, t)\right](p, s, k) \\
& =\iiint_{0}^{\infty} \exp [-p x] \exp [-s y] \exp [-k t] \exp [-a x] \\
& \quad \times \exp [-b y] \exp [-c t] f(x, y, t) d x d y d t \\
& \int_{0}^{\infty} \exp [-p x] \exp [-a x] \\
& \quad \times\left(\iint_{0}^{\infty} \exp [-s y] \exp [-k t] \exp [-b y]\right. \\
& \quad \times \exp [-c t] f(x, y, t) d t d y) d t .
\end{aligned}
$$

Note that the integral inside the bracket satisfies the properties of the double Laplace transform and is given as [11]

$$
\begin{gathered}
\left(\iint_{0}^{\infty} \exp [-s y] \exp [-k t] \exp [-b y] \exp [-c t]\right. \\
\quad \times f(x, y, t) d t d y)=F(x, s+b, k+d)
\end{gathered}
$$

Thus

$$
\begin{gathered}
\int_{0}^{\infty} \exp [-p x] \exp [-a x] F(x, s+b, k+d) d t \\
\quad=F(p+a, s+b, k+d),
\end{gathered}
$$

and this completes the proof.

(ii) The following can also be observed:

$$
\frac{1}{\alpha \beta \gamma} F\left(\frac{p}{\alpha}, \frac{s}{\beta}, \frac{k}{\gamma}\right)=L_{x, y, t}[f(\alpha x, \beta y, \gamma t)](p, s, k) .
$$

We will present the proof

$$
\begin{aligned}
& L_{x, y, t}[f(\alpha x, \beta y, \gamma t)](p, s, k) \\
& =\iiint_{0}^{\infty} \exp [-p x] \exp [-s y] \exp [-k t] \\
& \quad \times f(\alpha x, \beta y, \gamma t) d x d y d t \\
& \int_{0}^{\infty} \exp [-p x]\left(\iint_{0}^{\infty} \exp [-s y] \exp [-k t]\right. \\
& \quad \times f(\alpha x, \beta y, \gamma t) d y d t) d x
\end{aligned}
$$

Note that the double integral inside the bracket satisfies the property of the double Laplace transform as [11]

$$
\begin{gathered}
\left(\iint_{0}^{\infty} \exp [-s y] \exp [-k t] f(\alpha x, \beta y, \gamma t) d y d t\right) \\
\quad=\frac{1}{\beta \gamma} F\left(\alpha x, \frac{s}{\beta}, \frac{k}{\gamma}\right) .
\end{gathered}
$$

Thus

$$
\begin{aligned}
L_{x, y, t} & {[f(\alpha x, \beta y, \gamma t)](p, s, k) } \\
& =\int_{0}^{\infty} \exp [-p x] \frac{1}{\beta \gamma} F\left(\alpha x, \frac{s}{\beta}, \frac{k}{\gamma}\right) d x \\
& =\frac{1}{\alpha \beta \gamma} F\left(\frac{p}{\alpha}, \frac{s}{\beta}, \frac{k}{\gamma}\right),
\end{aligned}
$$

and this completes the proof.

(iii) The following property can also be observed:

$$
\begin{aligned}
& \frac{\partial^{n+m+v}[F(p, s, k)]}{\partial p^{n} \partial s^{n} \partial k^{v}} \\
& \quad=L_{x, y, t}\left[(-1)^{n+m+v} x^{n} y^{m} t^{v} f(x, y, t)\right](p, s, k) .
\end{aligned}
$$

We will present the proof

$$
\begin{aligned}
F(p, s, k)=\iiint_{0}^{\infty} & \exp [-p x] \exp [-s y] \exp [-k t] \\
& \times f(x, y, t) d x d y d t
\end{aligned}
$$

Then,

$$
\begin{aligned}
\frac{\partial^{n+m+v}[F(p, s, k)]}{\partial p^{n} \partial s^{n} \partial k^{v}} & \\
=\frac{\partial^{n+m+v}}{\partial p^{n} \partial s^{n} \partial k^{v}}\left(\iiint_{0}^{\infty}\right. & \exp [-p x] \exp [-s y] \\
& \times \exp [-k t] f(x, y, t) d x d y d t) .
\end{aligned}
$$

Now making use of the convergence properties of the improper integral involved, we can interchange the operation of differentiation and integration and differentiate with 
respect to $p, s$, and $k$ under the integral sign. Thus, we arrive at the following expression:

$$
\begin{aligned}
& \frac{\partial^{n+m+v}[F(p, s, k)]}{\partial p^{n} \partial s^{n} \partial k^{v}} \\
& =\frac{\partial^{n}}{\partial p^{n}} \int_{0}^{\infty} \exp [-p x] \\
& \quad \times\left(\frac{\partial^{m+v}}{\partial s^{n} \partial k^{v}} \iint_{0}^{\infty} \exp [-s y] \exp [-k t]\right. \\
& \quad \times f(x, y, t) d y d t) d x .
\end{aligned}
$$

Note that the expression in the bracket satisfies the property of the double Laplace transform as [11]

$$
\begin{gathered}
\frac{\partial^{m+v}}{\partial s^{n} \partial k^{v}} \iint_{0}^{\infty} \exp [-s y] \exp [-k t] f(x, y, t) d y d t \\
=L_{y, t}\left[(-1)^{m+v} y^{m} t^{v} f(x, y, t)\right](s, k) .
\end{gathered}
$$

Thus

$$
\begin{aligned}
& \frac{\partial^{n+m+v}[F(p, s, k)]}{\partial p^{n} \partial s^{n} \partial k^{v}} \\
& =\frac{\partial^{n}}{\partial p^{n}} \int_{0}^{\infty} \exp [-p x] \\
& \quad \times\left(L_{y, t}\left[(-1)^{m+v} y^{m} t^{v} f(x, y, t)\right](s, k)\right) d x .
\end{aligned}
$$

And finally, we obtain

$$
\begin{aligned}
& \frac{\partial^{n+m+v}[F(p, s, k)]}{\partial p^{n} \partial s^{n} \partial k^{v}} \\
& \quad=L_{x, y, t}\left[(-1)^{n+m+v} x^{n} y^{m} t^{v} f(x, y, t)\right](p, s, k),
\end{aligned}
$$

and this completes the proof.

Now using the previous three properties, we will show the proof of Theorem 5 .

Proof of Theorem 5. Let us define the set of functions depending on parameters $m, n$, and $v$ as

$$
h_{m, n, v}(x, y, t)=\frac{m^{m+1} n^{n+1} v^{v+1}}{m ! n ! v !} x^{m} y^{n} t^{v} e^{-m x-n y-v t} .
$$

It worth noting that the previous function is a kind of threedimensional density of probability, and it therefore follows that

$$
\iiint_{0}^{\infty} h_{m, n, v}(x, y, t) d x d y d t=1 .
$$

In addition of this, we will have that

$$
\lim _{\substack{m \rightarrow \infty \\ n \rightarrow \infty \\ v \rightarrow \infty}} \iiint_{0}^{\infty} h_{m, n, v}(x, y, t) \psi(x, y, t) d x d y d t=\psi(1,1,1),
$$

where $\psi(x, y, t)$ is any continuous function. Let $\Psi(p, s, k)$ denote the triple Laplace transform of the continuous function $\psi(x, y, t)$. However, if one defines the function $M(x, y, t)=f(x \alpha, y \beta, t \gamma)$, making use of the second property established in (29), we arrive at the following:

$$
\frac{1}{\alpha \beta \gamma} F\left(\frac{p}{\alpha}, \frac{s}{\beta}, \frac{k}{\gamma}\right)=L_{x, y, t}[f(\alpha x, \beta y, \gamma t)](p, s, k) .
$$

Here if one applies the third property, in particular by replacing $p=m / x, s=n / y, k=v / t$ as follows:

$$
\begin{aligned}
L_{x y t}(M(x, y, t))=\frac{1}{\alpha \beta \gamma} F\left(\frac{p}{\alpha}, \frac{s}{\beta}, \frac{k}{\gamma}\right), \\
=\frac{\partial^{n+m+v}\left[L_{x y t}(M(x, y, t))\right]}{\partial p^{n} \partial s^{n} \partial k^{v}} \\
=\frac{\partial^{n+m+v}[(1 / \alpha \beta \gamma) F(p / \alpha, s / \beta, k / \gamma)]}{\partial p^{n} \partial s^{n} \partial k^{v}} \\
\alpha^{m+1} \beta^{n+1} \gamma^{v+1} \\
\quad \times \frac{\partial^{n+m+v}[F(p / \alpha, s / \beta, k / \gamma)]}{\partial p^{n} \partial s^{n} \partial k^{v}} .
\end{aligned}
$$

Now let us put $\psi(x, y, t)=e^{-p x-s y-k t} M(x, y, t)$. Now if we make use of (38), we obtain the following

$$
\begin{aligned}
& \psi(1,1,1)=e^{-p-s-k} M(1,1,1)=e^{-p-s-k} f(\alpha, \beta, \gamma) \\
& =\lim _{\substack{m \rightarrow \infty \\
n \rightarrow \infty \\
v \rightarrow \infty}} \frac{m^{m+1} n^{n+1} v^{v+1}}{m ! n ! v !} \iiint_{0}^{\infty} x^{m} y^{n} t^{v} e^{-p x-s y-k t} \\
& \times e^{-m x-n y-v t} \Psi(x, y, t) d x d y d t \\
& =\lim _{\substack{m \rightarrow \infty \\
n \rightarrow \infty \\
v \rightarrow \infty}} \frac{m^{m+1} n^{n+1} v^{v+1}}{m ! n ! v !} L_{x y t}\left[x^{m} y^{n} t^{v} e^{-m x-n y-v t} \Psi(x, y, t)\right] \text {. }
\end{aligned}
$$

Now taking into account properties (i) and (ii), (42) together with the function $M(x, y, t)$, we arrive at the following:

$$
\begin{aligned}
& L_{x y t}\left[x^{m} y^{n} t^{v} e^{-m x-n y-v t} \Psi(x, y, t)\right] \\
& =(-1)^{m+n+v} \frac{\partial^{n+m+v}\left[L_{x y t}\left(e^{-m x-n y-k t} \Psi(x, y, t)\right)(p, s, k)\right]}{\partial p^{n} \partial s^{n} \partial k^{v}} \\
& =(-1)^{m+n+v} \frac{1}{\alpha^{m} \beta^{n} \gamma^{v}} \\
& \quad \times \frac{\partial^{n+m+v}\left[L_{x y t}(\Psi(x, y, t))(p+m, s+n, k+v)\right]}{\partial p^{n} \partial s^{n} \partial k^{v}} \\
& =(-1)^{m+n+v} \frac{1}{\alpha^{m} \beta^{n} \gamma^{v}} \quad\left[L_{x y t}(f(\alpha x, \beta y, \gamma t))\right. \\
& \left.\quad \times\left(\left(\partial^{n+m+v}\left[\frac{p+m}{\alpha}, \frac{s+n}{\beta}, \frac{k+v}{\gamma}\right)\right]\right)\left(\partial p^{n} \partial s^{n} \partial k^{v}\right)^{-1}\right)
\end{aligned}
$$




$$
\begin{aligned}
& =(-1)^{m+n+v} \\
& \quad \times \frac{1}{\alpha^{m} \beta^{n} \gamma^{v}} \frac{\partial^{n+m+v}[F((p+m) / \alpha,(s+n) / \beta,(k+v) / \gamma)]}{\partial p^{n} \partial s^{n} \partial k^{v}} .
\end{aligned}
$$

Now observe that from (44) with the fact that $f(\alpha, \beta, t)=$ $\psi(1,1,1) e^{p+s+k}$, we arrive at the following:

$$
\begin{aligned}
f(\alpha, \beta, t) & e^{p+s+k} \lim _{\substack{m \rightarrow \infty \\
n \rightarrow \infty \\
v \rightarrow \infty}} \frac{m^{m+1} n^{n+1} v^{v+1}}{m ! n ! v !} \\
& \times\left(\frac{m}{\alpha}\right)^{m+1}\left(\frac{n}{\beta}\right)^{n+1}\left(\frac{v}{\gamma}\right)^{v+1} \\
& \times \frac{\partial^{n+m+v}[F((p+m) / \alpha,(s+n) / \beta,(k+v) / \gamma)]}{\partial p^{n} \partial s^{n} \partial k^{v}} .
\end{aligned}
$$

The previously mentioned is true for any $p, s, k$ in the complete space, in particular, for $p=0, s=0, k=0$, and in this case Theorem 5 is covered.

\section{Application to Third-Order Partial Differential Equation}

In this section, we present the application of this operator for solving some kind of third-order partial differential equations.

Example 1. consider the following third-order partial differential equation:

$$
\partial_{x y t} u(x, y, t)+u(x, y, t)=0 .
$$

The previous equation is called the Mboctara equation and is subjected to the following boundaries and initial conditions:

$$
\begin{array}{ll}
u(x, y, 0)=e^{x+y}, & u(x, 0, t)=e^{x-t}, \\
u(0, y, t)=e^{y-t}, & u(x, y, 1)=e^{x+y-1} .
\end{array}
$$

Now applying the triple Laplace transform on both sides of (46), we obtain the following:

$$
p s k U(p, s, k)+U(p, s, k)=G(p, s, k) .
$$

Here

$$
\begin{aligned}
G(p, s, k)= & p s U(p, s, 0)+p s U(p, 0, k)-p U(p, 0,0) \\
& +s k U(0, s, k)-s U(0, s, 0)-k U(0,0, k) \\
& +U(0,0,0) .
\end{aligned}
$$

Factorising the right side of equation (49), we obtain the following:

$$
U(p, s, k)=\frac{G(p, s, k)}{1+p s k}
$$

Now applying the inverse triple Laplace transform on the previous equation we obtain the following solution:

$$
u(x, y, t)=L_{x y t}^{-1}\left[\frac{G(p, s, k)}{1+p s k}\right]=e^{x+y-t} .
$$

This is the exact solution for Mboctara equation.

Example 2. Let us consider the following nonhomogeneous Mboctara equation

$$
\partial_{x y t} u(x, y, t)+u(x, y, t)=-e^{x-2 y+t}
$$

subjected to the following initial and boundaries conditions:

$$
\begin{gathered}
u(x, 0,0)=e^{x}, \quad \partial_{t} u(x, 0, t)=e^{x+t}, \quad \partial_{x} u(x, 0, t)=e^{x+t}, \\
u(0,0,0)=1, \quad u(x, 0.5, t)=e^{x+t-1} .
\end{gathered}
$$

Now applying the triple Laplace transform on both sides of (52), we obtain the following:

$$
\begin{aligned}
\operatorname{psk} U & (p, s, k)+U(p, s, k) \\
& =G(p, s, k)-\frac{1}{(1+p)(2+s)(1+k)} .
\end{aligned}
$$

Factorising the right side of (54), we obtain the following:

$$
U(p, s, k)=\frac{G(p, s, k)-1 /(1+p)(2+s)(1+k)}{1+p s k}
$$

Now applying the inverse triple Laplace transform on the previous equation, we obtain the following solution

$$
\begin{aligned}
u(x, y, t) & =L_{x y t}^{-1}\left[\frac{G(p, s, k)-1 /(1+p)(2+s)(1+k)}{1+p s k}\right] \\
& =e^{x-2 y+t} .
\end{aligned}
$$

This is the exact solution for nonhomogeneous Mboctara equation.

Example 3. Let us consider the following nonhomogeneous Mboctara equation

$$
\begin{aligned}
\partial_{x y t} u(x, y, t)+u(x, y, t)= & \cos (x) \cos (y) \cos (-t) \\
& -\sin (x) \sin (y) \sin (-t),
\end{aligned}
$$

subjected to the following initial and boundaries conditions:

$$
\begin{gathered}
u(x, y, 0)=\cos (x) \cos (y), \\
\partial_{t} u(x, y, 0)=\partial_{x} u(0, y, t)=\partial_{y} u(x, 0, t)=0, \\
u\left(x, \frac{\pi}{2}, t\right)=u\left(x, y, \frac{\pi}{2}\right)=u\left(\frac{\pi}{2}, y, t\right)=0 .
\end{gathered}
$$


TABLE 1: Table of triple Laplace transform for some function of three variables.

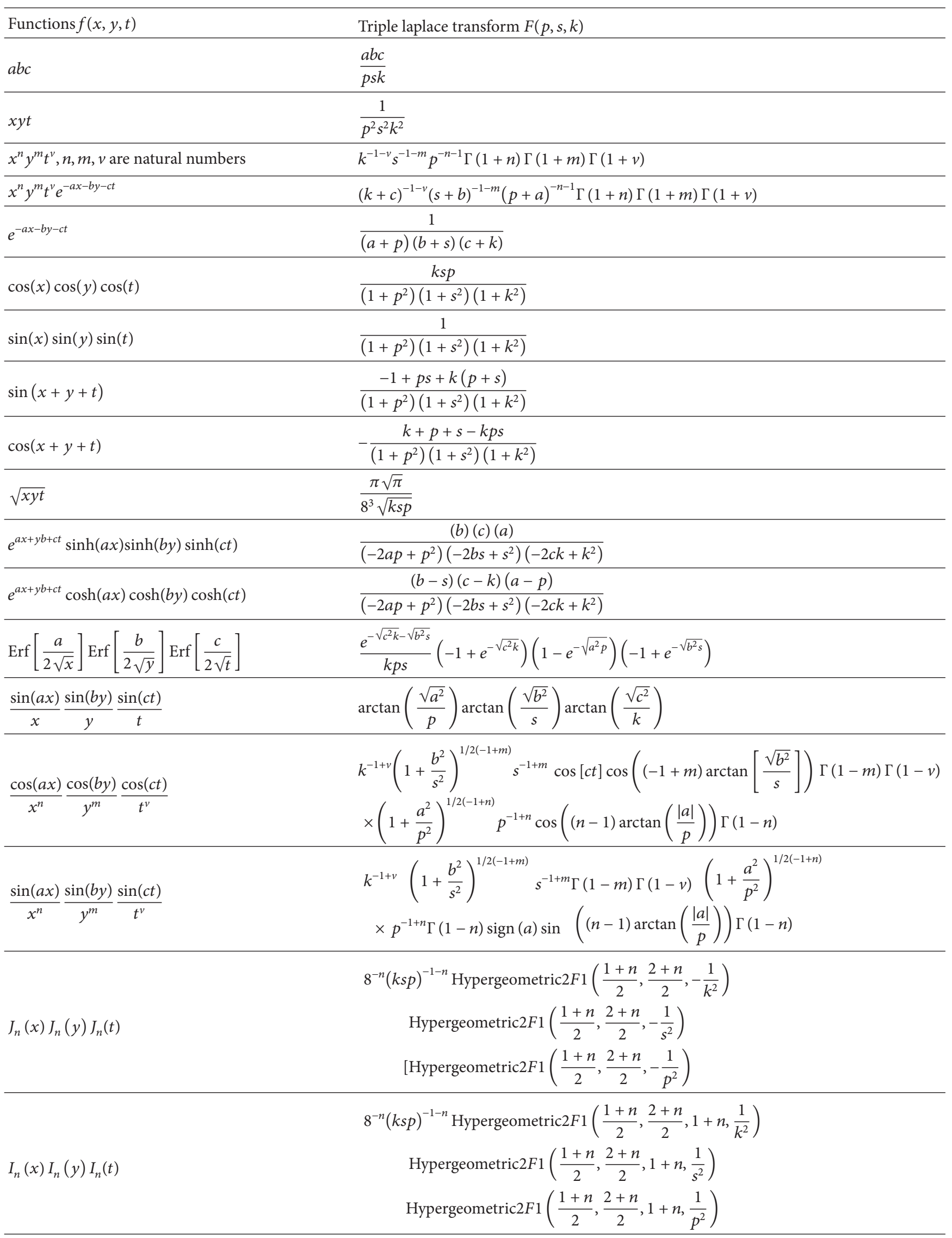




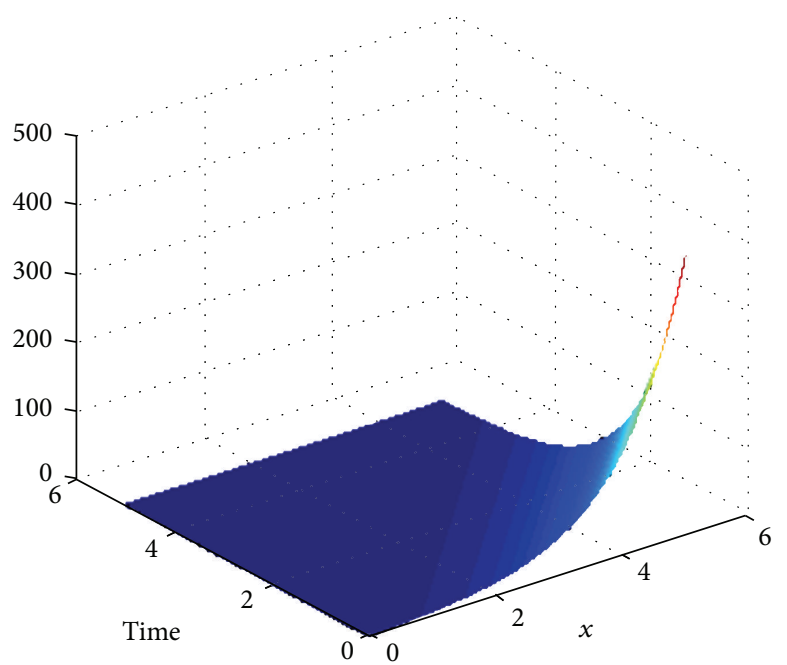

(a)

Exact solution of the nonhomogeneous Mboctara equation (4.6)

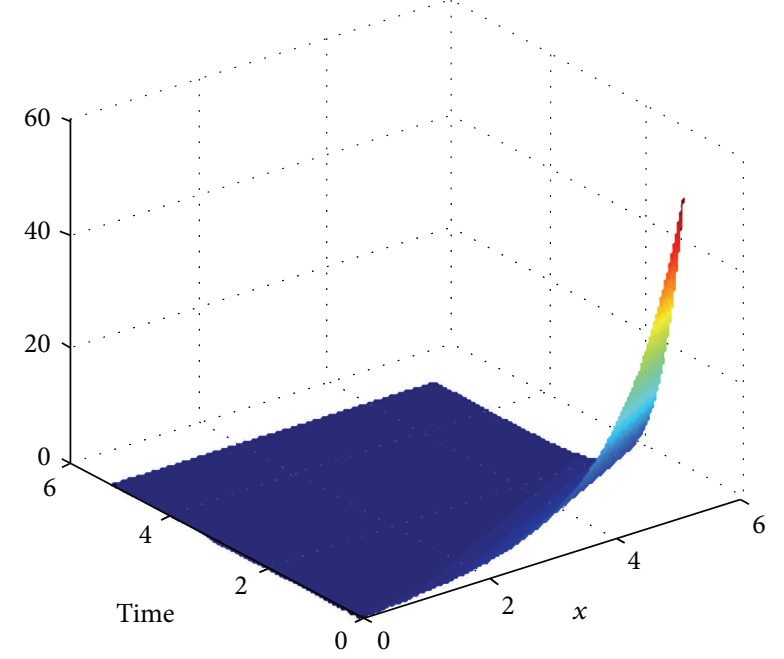

(c)

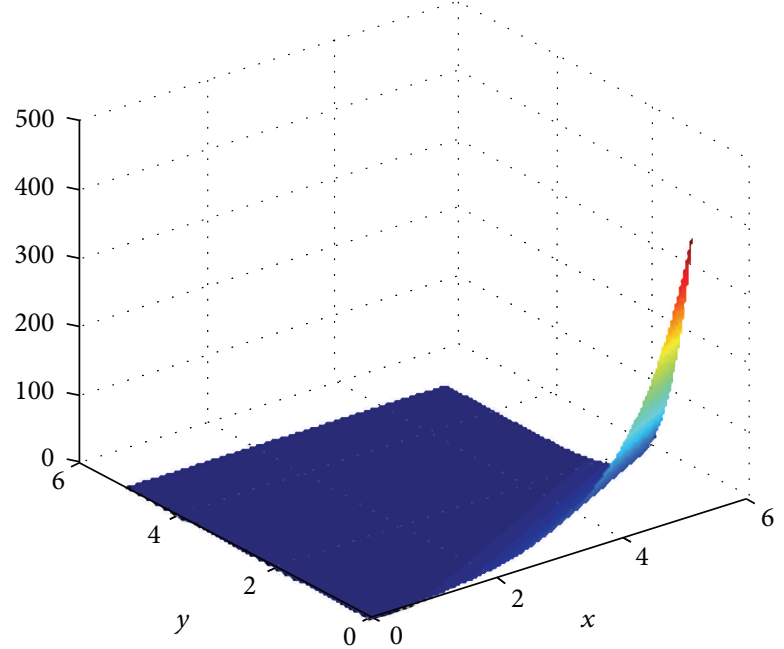

(b)

Exact solution of the nonhomogeneous Mboctara equation (4.11)

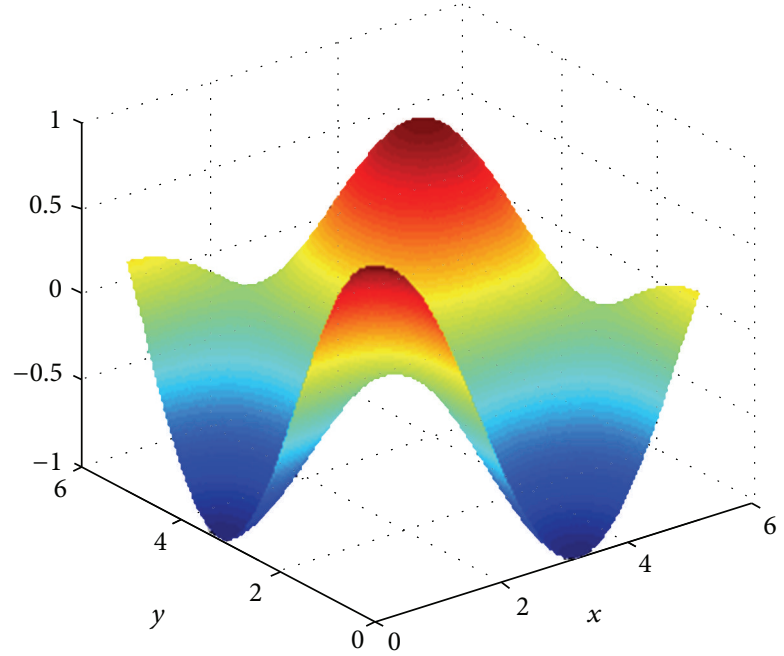

(d)

FIGURE 1: Numerical simulation of the exact solutions of the Homogeneous and non-homogeneous Mboctara equations.

Now applying the triple Laplace transform on both sides of (57), we obtain the following:

$$
\begin{aligned}
p s k U & (p, s, k)+U(p, s, k) \\
= & G(p, s, k)+\frac{k s p}{\left(1+p^{2}\right)\left(1+s^{2}\right)\left(-1+k^{2}\right)} \\
& -\frac{1}{\left(1+p^{2}\right)\left(1+s^{2}\right)\left(-1+k^{2}\right)} .
\end{aligned}
$$

Factorising the right side of (59), we obtain the following:

$$
\begin{aligned}
U(p, s, k)= & \frac{G(p, s, k)}{1+p s k}+\frac{k s p /\left(1+p^{2}\right)\left(1+s^{2}\right)\left(-1+k^{2}\right)}{1+p s k} \\
& -\frac{1 /\left(1+p^{2}\right)\left(1+s^{2}\right)\left(-1+k^{2}\right)}{1+p s k}
\end{aligned}
$$


Now applying the inverse triple Laplace transform on the previous equation, we obtain the following solution:

$$
\begin{aligned}
& u(x, y, t) \\
& =L_{x y t}^{-1}\left[\frac{G(p, s, k)}{1+p s k}+\frac{k s p /\left(1+p^{2}\right)\left(1+s^{2}\right)\left(-1+k^{2}\right)}{1+p s k}\right. \\
& \left.-\frac{1 /\left(1+p^{2}\right)\left(1+s^{2}\right)\left(-1+k^{2}\right)}{1+p s k}\right] \\
& =\cos (x) \cos (y) \cos (-t) .
\end{aligned}
$$

This is the exact solution for nonhomogeneous Mboctara equation.

Example 4. consider the following nonlinear nonhomogeneous with variable coefficient Mboctara equation:

$$
\begin{aligned}
& e^{x+y+t} \partial_{x y t} u(x, y, t)-3 u^{2}(x, y, t)+e^{x+y+t} u(x, y, t) \\
& =e^{2 x+2 y+2 t}, \\
& u_{x}(x, y, 0)=e^{x+y}, \quad u(0,0,0)=1, \\
& u(1,0,0)=e, \quad \partial_{x y t} u(0,0,0)=1 .
\end{aligned}
$$

Now applying the triple Laplace transform on both sides of (62) and then using the properties of the triple Laplace transform and after factorising as in the previous examples and taking the inverse triple Laplace transform, we obtain the following as an exact solution of this type of Mboctara equation:

$$
u(x, y, t)=e^{x+y+t}
$$

The numerical simulations of the exact solutions of the Mboctara equation are depicted in Figure 1(a) (4.1), Figure 1(b) (4.6), Figure 1(c) (4.6) and Figure 1(d) (4.11), respectively.

\section{Triple Laplace Transform of Some Functions of Three Variables}

In this section, we examine the triple Laplace transform of some functions in Table 1:

$$
\begin{aligned}
L_{x y t}\left(Y_{n}(x)\right. & \left.Y_{n}(y) Y_{n}(t)\right) \\
= & 8^{-n}(k s p)^{-1-n} \operatorname{Csc}^{3}[n \pi] \\
& \times\left(-\left(4 k^{2}\right)^{n} \text { Hypergeometric } 2 F 1\right. \\
& \times\left(\frac{1+n}{2}, \frac{2+n}{2}, 1-n,-\frac{1}{k^{2}}\right) \\
& +\cos (n \pi) \text { Hypergeometric } 2 F 1 \\
& \left.\times\left(\frac{1+n}{2}, \frac{2+n}{2}, 1+n,-\frac{1}{k^{2}}\right)\right)
\end{aligned}
$$

$$
\begin{aligned}
\times(- & \left(4 s^{2}\right)^{n} \text { Hypergeometric } 2 F 1 \\
& \times\left(\frac{1+n}{2}, \frac{2+n}{2}, 1-n,-\frac{1}{s^{2}}\right) \\
& +\cos (n \pi) \text { Hypergeometric } 2 F 1 \\
& \left.\times\left(\frac{1+n}{2}, \frac{2+n}{2}, 1+n,-\frac{1}{s^{2}}\right)\right) \\
\times(- & \left(4 p^{2}\right)^{n} \text { Hypergeometric } 2 F 1 \\
& \times\left(\frac{1+n}{2}, \frac{2+n}{2}, 1-n,-\frac{1}{p^{2}}\right) \\
+ & \cos (n \pi) \text { Hypergeometric } 2 F 1 \\
\times & \left.\left(\frac{1+n}{2}, \frac{2+n}{2}, 1+n,-\frac{1}{p^{2}}\right)\right) .
\end{aligned}
$$

\section{Conclusion}

This work presents the definition of the triple Laplace transform. Some triple Laplace transform is presented in Table 1. Some theorems and properties of this new relatively new operator are presented. Applications of the new operator, for solving some kind of third-order partial differential equations called Mboctara equation, are presented. Numerical solutions of the Mboctara equation are given.

\section{References}

[1] G. L. Lamb Jr., Introductory Applications of Partial Differential Equations with Emphasis on Wave Propagation and Diffusion, John Wiley \& Sons, New York, NY, USA, 1995.

[2] U. T. Myint, Differential Equations of Mathematical Physics, American Elsevier, New York, NY, USA, 1980.

[3] C. Constanda, Solution Techniques for Elementary Partial Differential Equations, Chapman \& Hall/CRC, New York, NY, USA, 2002.

[4] D. G. Duffy, Transform Methods for Solving Partial Differential Equations, CRC Press, New York, NY, USA, 2004.

[5] A. Babakhani and R. S. Dahiya, "Systems of multi-dimensional Laplace transforms and a heat equation," in Proceedings of the 16th Conference on Applied Mathematics, vol. 7 of ElectronicJournal of Differential Equations, pp. 25-36, University of Central Oklahoma, Edmond, Okla, USA, 2001.

[6] Y. A. Brychkov, H. -J. Glaeske, A. P. Prudnikov, and V. K. Tuan, Multidimensional Integral Transformations, Gordon and Breach Science Publishers, Philadelphia, Pa, USA, 1992.

[7] A. Atangana and A. Kilicman, "A possible generalization of acoustic wave equation using the concept of perturbed derivative order," Mathematical Problems in Engineering, vol. 2013, Article ID 696597, 6 pages, 2013.

[8] A. K1lıçman and H. Eltayeb, "A note on the classifications of hyperbolic and elliptic equations with polynomial coefficients," Applied Mathematics Letters, vol. 21, no. 11, pp. 1124-1128, 2008. 
[9] H. Eltayeb, A. Kılıçman, and P. Ravi Agarwal, "An analysis on classifications of hyperbolic and elliptic PDEs," Mathematical Sciences, vol. 6, article 47, 2012.

[10] H. Eltayeb and A. Kılıçman, "A note on double laplace transform and telegraphic equations," Abstract and Applied Analysis, vol. 2013, Article ID 932578, 6 pages, 2013.

[11] A. Kılıçman and H. E. Gadain, "On the applications of Laplace and Sumudu transforms," Journal of the Franklin Institute, vol. 347, no. 5, pp. 848-862, 2010.

[12] R. P. Kanwal, Generalized Functions Theory and Applications, Birkhäauser, Boston, Mass, USA, 2004. 


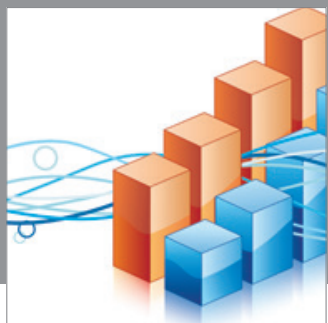

Advances in

Operations Research

mansans

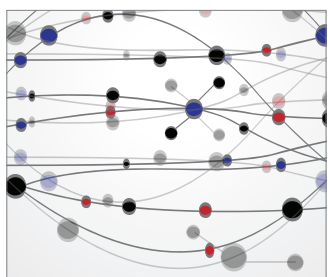

The Scientific World Journal
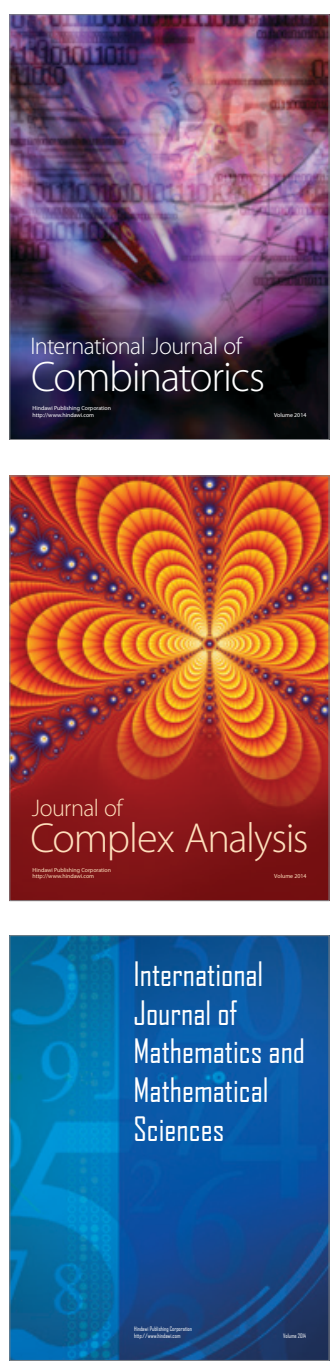
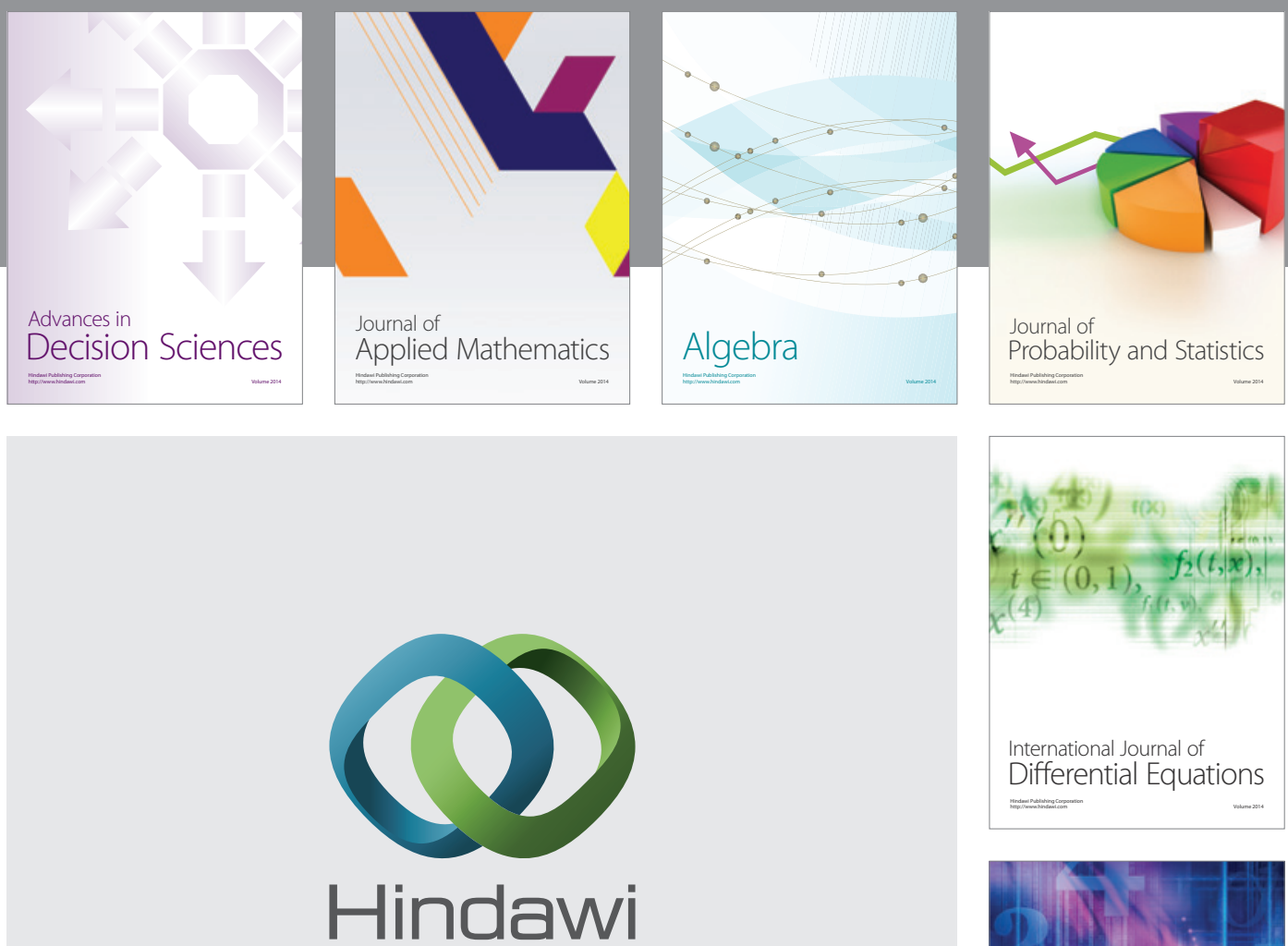

Submit your manuscripts at http://www.hindawi.com
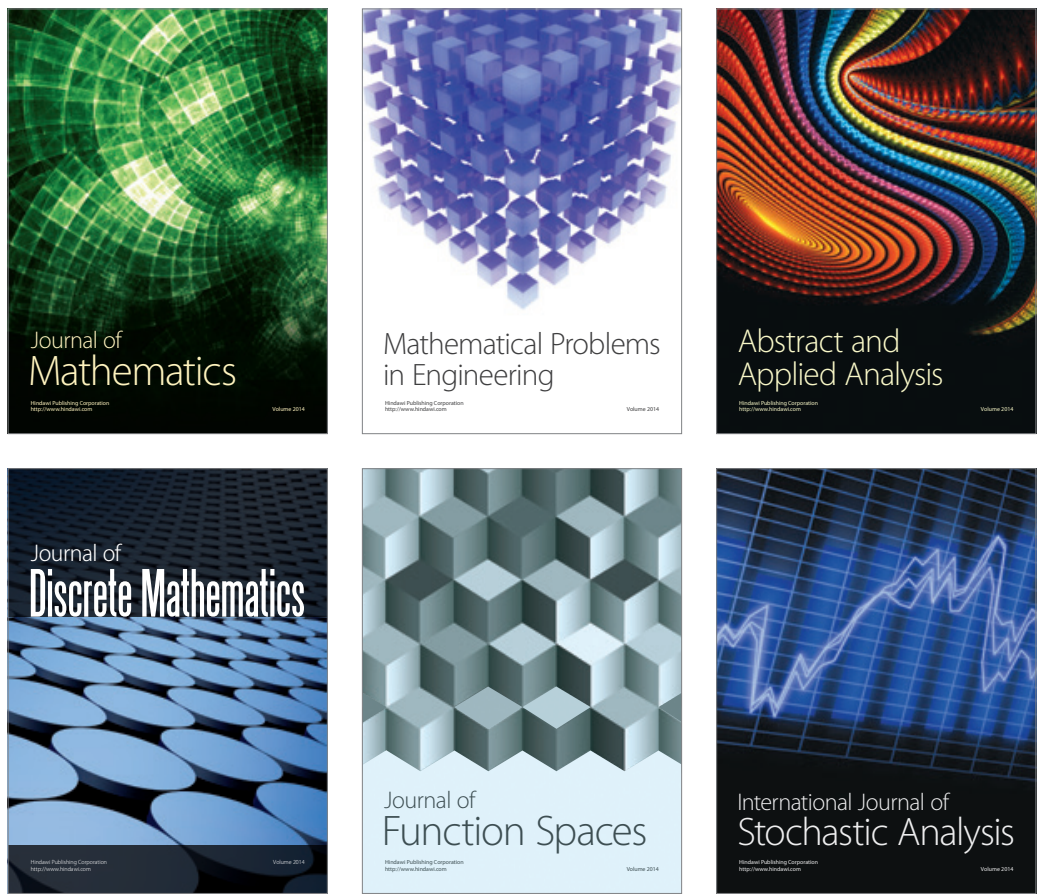

Journal of

Function Spaces

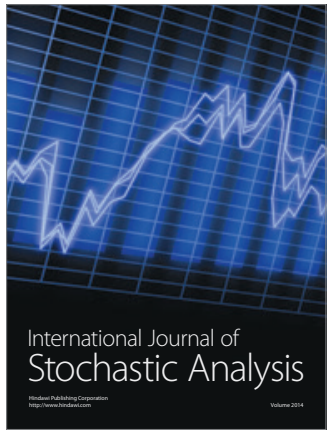

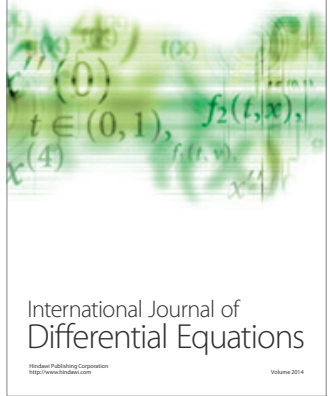
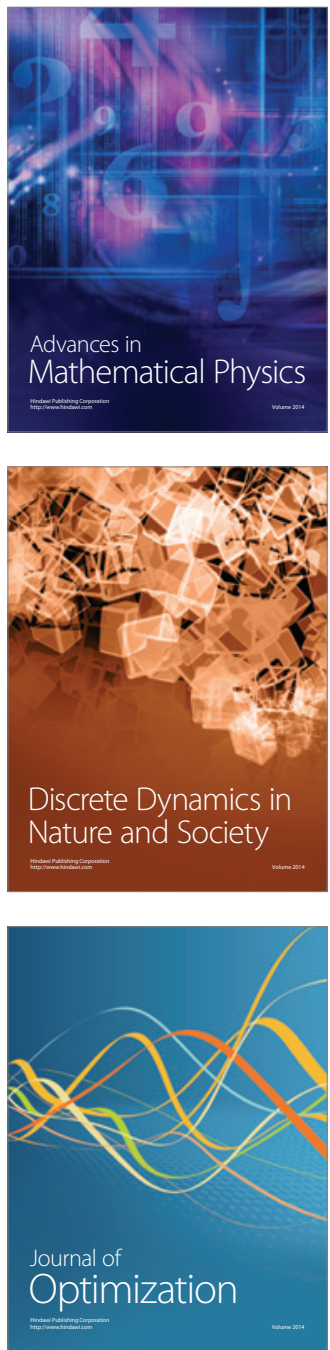